\title{
Some observations on the Furstenberg topological space
}

Rezsô L. Lovas and István Mező

Rezső L. Lovas obtained his Ph.D. in 2006 at the University of Debrecen, Hungary. He is now a senior lecturer at the same university. His main field of interest is differential geometry.

István Mezô obtained his Ph.D. in 2010 at the University of Debrecen, Hungary. He now works as a research professor at the Nanjing University of Information Science and Technology, P. R. China. His research mainly concentrates on special function theory, analytic number theory, and enumerative combinatorics.

\section{Introduction}

In $1955 \mathrm{H}$. Furstenberg introduced a simple topology on the set of rational integers to prove the infiniteness of the set of primes [1, 6, 11]. In this topology, a subset $A$ of $\mathbb{Z}$ is open if and only if each of its points is contained in $A$ together with an infinite arithmetic progression. We present the precise definition in Section 2. This topological space gives very good, nontrivial, though elementary examples and counterexamples for many topological concepts, see for example the book of Steen and Seebach [13, pp. 80-81].

In this paper we study some of its interesting topological properties and its metrizations. Among others, in Section 3 we give a proof for its non-compactness which does not use a metrization, and we characterize convergent sequences.

From Urysohn's metrizability theorem it can be easily deduced that the Furstenberg topology is metrizable (see, e.g., [7]). Urysohn's theorem, however, does not provide an explicit

Im IX. Buch von Euklids Elementen findet sich der heute wohlbekannte zahlentheoretische Beweis, dass die Folge der Primzahlen nicht abbricht. Im Jahre 1955 gab Hillel Fürstenberg einen überraschenden topologischen Beweis für eben diesen Satz. Zahlentheorie und Topologie scheinen auf den ersten Blick völlig unabhängige Gebiete der Mathematik zu sein. Doch gibt Fürstenbergs Topologie auf der Menge der ganzen Zahlen einen engen Zusammenhang zwischen diesen zwei fernen Gebieten. Aus diesem Grund ist sie für viele Mathematikerinnen und Mathematiker so attraktiv. In der vorliegenden Arbeit werden verschiedene interessante Aspekte dieser Topologie betrachtet. Insbesondere wird auch eine metrische Vervollständigung vorgestellt. 
method of finding a metric which induces a given topology. Therefore, in Section 4 we present three different translation invariant metrics on $\mathbb{Z}$ inducing the Furstenberg topology. The first of these were originally proposed by the present authors in [10], the second one by J. Ferry on an Internet forum [5], and the third one appears here for the first time. In this we make use of the so-called factorial number system, to which we also give a brief introduction.

In Section 4 we also construct the metrical completion of this third metrization. The completion of a certain metrization of a topological space is not a natural construction in general, since the completions of homeomorphic metric spaces are not necessarily homeomorphic to each other. Our completion is still natural in a certain sense, since, as we shall see, if two different translation invariant metrics on $\mathbb{Z}$ generate the Furstenberg topology, then their completions are automatically uniformly equivalent.

\section{The Furstenberg topology}

Throughout this paper, we shall denote the set of natural numbers (positive integers) by $\mathbb{N}$ and the set of integers by $\mathbb{Z}$.

Let us define the Furstenberg topology $\mathcal{T}$ on $\mathbb{Z}$ as follows. If $a, b \in \mathbb{Z}, a \neq 0$, then we call the set

$$
a \mathbb{Z}+b=\{a k+b: k \in \mathbb{Z}\}
$$

an (infinite) arithmetic progression. Let $\mathcal{T}$ be the topology generated by all sets of this form. Thus, a general open set is a (possibly empty) union of arithmetic progressions. The topological space $(\mathbb{Z}, \mathcal{T})$ is called the Furstenberg space.

We collect some useful basic facts about the space $(\mathbb{Z}, \mathcal{T})$ for further reference.

- A point $p$ is an inner point in a set $A \subset \mathbb{Z}$ if $A$ contains an arithmetic progression containing the point $p$. In other words, $p \in A$ is an inner point if there are some $a, b \in \mathbb{Z}, a \neq 0$ such that $p \in a \mathbb{Z}+b \subset A$.

- We saw above that a set is open if and only if it is a union of arithmetic progressions. This immediately yields that a set consisting only of positive (or negative) elements cannot be open. Similarly, a finite nonempty set cannot be open.

- A set of the form $a \mathbb{Z}+b$ is closed as well. This follows from the fact that its complement is the union of the arithmetic progressions

$$
a \mathbb{Z}+b+1, \ldots, a \mathbb{Z}+b+a-1
$$

(provided that $a>0$ )

\section{Some additional topological facts in $(\mathbb{Z}, \mathcal{T})$}

Now we go a bit deeper into the topological structure of the Furstenberg space and we consider some of its less trivial properties. 


\subsection{Closedness}

We saw above that a finite nonempty set cannot be open. Now the question arises whether every finite set is closed or not. The answer is yes. Even singletons are closed.

Theorem 1. The singletons $\{c\}$ are closed sets.

Proof. We saw above that the arithmetic progressions are closed sets. We show that

$$
\{c\}=\bigcap_{a=1}^{\infty}(a \mathbb{Z}+c) .
$$

Suppose that the right-hand side contains an element $c+d$ or $c-d$ with $d>0$. This element is not contained in $2 d \mathbb{Z}+c$, which is a contradiction. Thus $\{c\}$ is an intersection of closed sets, thus it is itself closed.

Remark 1. Later we shall see that $(\mathbb{Z}, \mathcal{T})$ is metrizable, so it is Hausdorff, from which it also follows that singletons are closed.

In the tiny literature of $(\mathbb{Z}, \mathcal{T})$ we can find some results on the closure of special sets. We cite a few of these results.

- The set of squarefree integers (excluding \pm 1 ) is closed [2, p. 720].

- The set of Mersenne numbers (of the form $2^{p}-1$, where $p$ is a prime) is closed [2, p. 720].

- The set of Fermat numbers $2^{2^{n}}+1(n \geq 0)$ is closed [2, p. 720].

- The closure of the set $\mathbb{P}$ of primes is $\mathbb{P} \cup\{-1,1\}[2$, p. 718].

- The closure of the set of Fibonacci numbers $F_{n}$ is $\left\{F_{n}: n \geq 0\right\} \cup\left\{(-1)^{n+1} F_{n}: n \geq\right.$ $0\}$. See [8] for the original proof and [3] for a nice generalization.

\subsection{Cofinite sets and denseness}

A set in $\mathbb{Z}$ is called cofinite if its complement is finite. We can easily see that cofinite sets different from $\mathbb{Z}$ cannot be closed. (If they were closed, their complements would be open, which is impossible.) Then the question arises: what is the closure of a cofinite set?

To answer this question we use the simple topological fact

$$
\operatorname{cl}(A)=\left(\operatorname{int}\left(A^{c}\right)\right)^{c},
$$

where cl denotes closure, int denotes interior and $c$ complement.

Theorem 2. The closure of a cofinite set is $\mathbb{Z}$. In other words, cofinite sets are dense in $\mathbb{Z}$.

Proof. Let $A$ be a cofinite set in $\mathbb{Z}$. Then $\mathbb{Z} \backslash A$ is finite, so it does not contain an inner point: $\operatorname{int}(\mathbb{Z} \backslash A)=\emptyset$. Then equality (1) yields that $\operatorname{cl}(A)=\mathbb{Z}$. 
Are there 'smaller' sets which are dense? We know by Theorem 1 that finite sets cannot be dense. Hence we have to look for infinite sets. A surprising but easy fact is that the nonnegative integers form a dense subset.

Example 1. The set $\mathbb{N}_{0}=\{0,1,2,3, \ldots\}$ is dense in $(\mathbb{Z}, \mathcal{T})$. Indeed, using again (1) we find

$$
\operatorname{cl}\left(\mathbb{N}_{0}\right)=\mathbb{Z} \backslash \operatorname{int}\left(\mathbb{Z} \backslash \mathbb{N}_{0}\right) .
$$

The set $\mathbb{Z} \backslash \mathbb{N}_{0}$ of negative integers does not contain any inner points, since it does not contain any arithmetic progressions. Hence

$$
\operatorname{cl}\left(\mathbb{N}_{0}\right)=\mathbb{Z} \backslash \operatorname{int}\left(\mathbb{Z} \backslash \mathbb{N}_{0}\right)=\mathbb{Z} \backslash \emptyset=\mathbb{Z} .
$$

Example 2. Now we generalize the previous example. If we omit finitely many elements from the set

$$
\{k, k+1, k+2, \ldots\} \quad \text { or } \quad\{\ldots,-k-2,-k-1,-k\} \text {, }
$$

the remaining set will be still dense.

One can ask whether every dense set has the form as in the previous example. The answer is no: there are dense sets of different form, as the next example shows.

Example 3. The set $N:=\left\{ \pm n^{2}: n \in \mathbb{N}\right\}$ does not contain an inner point, since there is no arithmetic progression which contains only square numbers, so $\mathbb{Z} \backslash N$ is dense.

\subsection{Compactness}

A topological space $X$ is said to be compact if any family $\left(P_{i}\right)_{i \in I}$ of open sets which cover $X$, which means that

$$
X=\bigcup_{i \in I} P_{i}
$$

has a finite subfamily which also covers $X$. An important equivalent condition is the following. A family $\left(P_{i}\right)_{i \in I}$ of sets satisfies the finite intersection property (fip) if the intersection of any finite subfamily $\left(P_{i_{1}}, \ldots, P_{i_{k}}\right)$ is nonempty. The space $X$ is compact if and only if for any family $\left(P_{i}\right)_{i \in I}$ of closed sets satisfying fip, the intersection of $\left(P_{i}\right)_{i \in I}$ itself is not empty:

$$
\bigcap_{i \in I} P_{i} \neq \emptyset
$$

See [12] for the proof. This simple result enables us to prove the non-compactness of $(\mathbb{Z}, \mathcal{T})$.

Theorem 3. The Furstenberg space $(\mathbb{Z}, \mathcal{T})$ is not compact.

Proof. We construct a family of closed sets which satisfies fip, but the intersection of all the family is empty. Let

$$
P_{i}=p_{i} \mathbb{Z}+i,
$$


where $p_{i}$ is the $i$ th prime number, so

$$
P_{1}=2 \mathbb{Z}+1, \quad P_{2}=3 \mathbb{Z}+2, \quad P_{3}=5 \mathbb{Z}+3, \ldots .
$$

Let us choose an arbitrary finite family of these closed sets:

$$
P_{i_{1}}, P_{i_{2}}, \ldots, P_{i_{k}} \text {. }
$$

The intersection of these is not empty if and only if there is an $n$ such that

$$
\begin{gathered}
n=p_{i_{1}} j_{1}+i_{1}, \\
n=p_{i_{2}} j_{2}+i_{2}, \\
\quad \vdots \\
n=p_{i_{k}} j_{k}+i_{k} .
\end{gathered}
$$

Such an integer $n$ surely exists by the Chinese Remainder Theorem. Hence $\left(P_{i}\right)_{i \in \mathbb{N}}$ satisfies fip. However,

$$
\bigcap_{i \in \mathbb{N}} P_{i}=\varnothing
$$

since in the opposite case there would exist an integer $n$ such that

$$
n=p_{k} j_{k}+k \quad(k \in \mathbb{N}) .
$$

Such an $n$ cannot be nonnegative, since $p_{k}>k$, so the least nonnegative $n$ satisfying the equality must be equal to $k$. To see that $n$ cannot be negative either, observe first that $p_{k}>2 k$ if $k \geq 5$. If $n$ is negative, then each $j_{k}$ is negative, thus

$$
n=p_{k} j_{k}+k \leq-p_{k}+k<-2 k+k=-k
$$

for each $k \geq 5$, which is also impossible.

Remark 2. The non-compactness of $(\mathbb{Z}, \mathcal{T})$ was proved in [7], too. However, that proof uses several properties of the Legendre symbols, and it is more complicated than the one presented here.

Later we shall see that $(\mathbb{Z}, \mathcal{T})$ is a metrizable space which can be metrized in such a way that it is not complete. From this it also follows that it cannot be compact.

\subsection{Convergent sequences in $(\mathbb{Z}, \mathcal{T})$}

Now we turn to the question of convergence, which is a very interesting one in the Furstenberg space.

A sequence $\left(a_{n}\right)_{n \in \mathbb{N}}$ converges to a point $a$ in a topological space $(X, \mathcal{T})$ if for any neighbourhood of $a$ (a set containing an open set which contains $a$ ) there is an $n_{0}$ such that this neighbourhood contains all of $a_{n}$ if $n>n_{0}$. 
First we study the sequences converging to 0 in $(\mathbb{Z}, \mathcal{T})$. The open neighbourhoods of 0 are the sets $k \mathbb{Z}(k \in \mathbb{N})$ and unions of these. If $k$ tends to infinity, then $k \mathbb{Z}$ has less and less elements. This shows that typical sequences converging to 0 in Furstenberg's sense tend to infinity in the ordinary sense. Does every sequence of this type converge to 0 ? The answer is no. If a sequence tending to infinity has infinitely many odd members, then it has infinitely many members which are not contained in the neighbourhood $2 \mathbb{Z}$ of 0 , thus it does not converge to 0 . This heuristic consideration shows that the members of a sequence converging to 0 have more and more divisors as the index grows. The following theorem describes this in a precise way.

Theorem 4. Let $\left(a_{n}\right)_{n \in \mathbb{N}}$ be a sequence of integers. Then $\lim _{n \rightarrow \infty} a_{n}=0$ if and only if, for every natural number $m$ there is an index $n_{0}$ such that $a_{n}$ is divisible by $m$ whenever $n>n_{0}$.

Since our topology is translation invariant, this theorem provides a characterization for all convergent sequences: the sequence $\left(a_{n}\right)_{n \in \mathbb{N}}$ converges to $c$ if and only if $a_{n}-c$ converges to 0 .

Note that it is not sufficient to suppose that the condition holds for each prime number in the previous theorem. For example, the sequence $a_{n}=p_{1} p_{2} \cdots p_{n}$ satisfies the condition for each prime number, however, none of its members is divisible by 4 , therefore it does not converge to zero.

Example 4. The members of the sequence $a_{n}=n$ ! are divisible by $m$ if $n \geq m$. Hence we have the rather surprising fact that

$$
\lim _{n \rightarrow \infty} n !=0
$$

Moreover, $n !+7 \rightarrow 7$ as $n \rightarrow \infty$.

Example 5. The members of the sequence

$$
a_{n}=\operatorname{lcm}(1,2, \ldots, n)
$$

are divisible by $m$ if $n \geq m$ (here lcm denotes the least common multiple). So

$$
\lim _{n \rightarrow \infty} \operatorname{lcm}(1,2, \ldots, n)=0 .
$$

Example 6. An example for a positive sequence converging to a negative number: let

$$
a_{n}=1 \cdot 1 !+2 \cdot 2 !+\cdots+n \cdot n ! \text {. }
$$

Then it follows by induction that $a_{n}=(n+1) !-1$, so

$$
\lim _{n \rightarrow \infty} a_{n}=-1
$$

Before studying the metrizability of the Furstenberg space, we mention that connectedness questions in this space were treated in detail by P. Szczuka [14]. A simple fact that the reader can easily check is that $(\mathbb{Z}, \mathcal{T})$ is totally disconnected, i.e., its only connected subsets are the empty set and the singletons (see also [10]). 


\section{Some metrics inducing the Furstenberg topology}

Using the above characterization of sequences converging to zero, we can easily find a metric which induces the Furstenberg topology. By definition, a metric induces a topology if all open balls with respect to the metric form a basis for the topology. Or, equivalently, a sequence converges to a point $a$ in the topology if and only if the distance of the members of the sequence from the point $a$ tends to zero.

We say that a function \|\|$: \mathbb{Z} \rightarrow \mathbb{R}$ is a norm on $\mathbb{Z}$ if it satisfies

(1) $\|n\| \geq 0$, and $\|n\|=0$ if and only if $n=0$,

(2) $\|n\|=\|-n\|$,

(3) $\|n+m\| \leq\|n\|+\|m\|$

for all $n, m \in \mathbb{Z}$. We shall only consider translation invariant metrics on $\mathbb{Z}$, which means that there is a norm \|\|$: \mathbb{Z} \rightarrow \mathbb{R}$ such that $d(n, m)=\|n-m\|$ for each $n, m \in \mathbb{Z}$. Conversely, if \|\| is an arbitrary norm, then $d(n, m):=\|n-m\|$ defines a translation invariant metric on $\mathbb{Z}$, and we also say that the topology on $\mathbb{Z}$ induced by $d$ is the topology induced by the norm.

One such norm can be the following:

$$
\|n\|_{L}:=\frac{1}{\max \{m \in \mathbb{N}: 1,2, \ldots, m \mid n\}} \quad \text { if } n \neq 0, \quad \text { and } \quad\|0\|_{L}:=0 .
$$

Thus, for example,

$$
\|24\|_{L}=\frac{1}{4}
$$

since 1,2,3, 4 divide 24 , but 5 not any more. One can easily see that $\|n\|_{L} \rightarrow 0$ if and only if $n \rightarrow 0$ in the Furstenberg topology. Now the metric $d: \mathbb{Z} \times \mathbb{Z} \rightarrow \mathbb{R}$ can be defined as

$$
d(n, m):=\|n-m\|_{L} .
$$

It can be easily shown that this metric induces the Furstenberg topology, see also the paper [10].

The above norm is not the only one which induces the Furstenberg topology. A norm on $\mathbb{Z}$ induces the Furstenberg topology if and only if it has the following property: a sequence converges to 0 with respect to the topology if and only if the norm of its general term tends to 0 .

Hence, one can define a different norm as follows. For a fixed integer $n$, let $D_{n}$ denote the set of all positive divisors of $n$, and let

$$
\|n\|_{F}:=\sum_{k \in \mathbb{N} \backslash D_{n}} 2^{-k}=1-\sum_{k \in D_{n}} 2^{-k} .
$$

This norm was introduced by J. Ferry in 2009 on an Internet forum [5]. Ferry gave only a heuristic proof that the function $d(n, m):=\|n-m\|_{F}$ is really a metric. 
The paper [7] deals with the properties of these two metrics. Among others, the authors show that these two metrics are metrics indeed, and they do induce the Furstenberg topology. Several additional interesting properties are also deduced in [7].

Now we introduce a new metric, numerically different from the above two, and based on the so-called factorial number system. For this metric we can easily construct the metric closure of $(\mathbb{Z}, \mathcal{T})$.

\subsection{The factorial number system}

In this system a number is represented in factorial base instead of base 10. The $i$ th radix is $i$ !, where the highest digit allowed is $i$. Hence every natural number $n$ can be represented as

$$
n=i_{1} \cdot 1 !+i_{2} \cdot 2 !+i_{3} \cdot 3 !+\cdots+i_{k} \cdot k ! \quad\left(0 \leq i_{j} \leq j\right),
$$

and this representation is unique (there is no other sequence $i_{1}, \ldots, i_{k}$ which results the same $n)$. For instance,

$$
349=1 \cdot 1 !+0 \cdot 2 !+2 \cdot 3 !+4 \cdot 4 !+2 \cdot 5 ! .
$$

See more on the factorial number system (FNS) in [9].

With the help of the factorial number system it is easy to give a necessary and sufficient condition for a sequence of positive integers to converge to 0 in the Furstenberg topology, which is the analogue of Theorem 4.

Theorem 5. Let $\left(a_{n}\right)_{n \in \mathbb{N}}$ be a sequence of positive integers with FNS representation

$$
a_{n}=i_{1, n} \cdot 1 !+\cdots+i_{k_{n}, n} \cdot k_{n} ! \quad(n \in \mathbb{N}) .
$$

Then $\left(a_{n}\right)_{n \in \mathbb{N}}$ converges to 0 in the Furstenberg topology if and only if for any positive integer $m$ there is an index $n_{0}$ such that $i_{m, n}=0$ holds for all $n>n_{0}$.

Proof. Suppose that $a_{n}$ converges to 0 . If $m \in \mathbb{N}$, then, by Theorem 4 , there is an index $n_{0}$ such that $(m+1) ! \mid a_{n}$ for all $n>n_{0}$. If the digits $i_{1, n}, \ldots, i_{m, n}$ are not all zero for such an $n$, then $a_{n}$ lies between two adjacent multiples of $(m+1)$ !, which contradicts $(m+1) ! \mid a_{n}$. Thus $i_{1, n}=\cdots=i_{m, n}=0$.

Conversely, suppose that the condition of the theorem is satisfied and $m \in \mathbb{N}$. Applying the condition separately to each $j=1, \ldots, m-1$ rather than to $m$ alone, we obtain that there is an index $n_{j}$ such that $i_{j, n}=0$ for all $n>n_{j}$. Now let $n_{0}:=\max \left\{n_{1}, \ldots, n_{m-1}\right\}$. If $n>n_{0}$, then $i_{1, n}=\cdots=i_{m-1, n}=0$, thus $m$ ! $\mid a_{n}$, which implies that $a_{n}$ is divisible by $1, \ldots, m$, and $a_{n}$ tends to 0 .

\subsection{A new metric based on FNS}

If the FNS representation of $n \in \mathbb{N}$ is

$$
n=i_{1} \cdot 1 !+\cdots+i_{k} \cdot k !
$$


then we define

$$
\|n\|:=\frac{1}{\min \left\{j \in \mathbb{N}: i_{j} \neq 0\right\}} .
$$

If $n$ is a negative integer, then $\|n\|:=\|-n\|$, and $\|0\|:=0$. Now the new metric $d: \mathbb{Z} \times \mathbb{Z} \rightarrow \mathbb{R}$ will be

$$
d(n, m):=\|n-m\| .
$$

By the symmetry of the Furstenberg topology with respect to 0 , a sequence $\left(a_{n}\right)_{n \in \mathbb{N}}$ of integers converges to 0 if and only if $\left(\left|a_{n}\right|\right)_{n \in \mathbb{N}}$ converges to 0 . Moreover, if $\left(a_{n}\right)_{n \in \mathbb{N}}$ converges to 0 , and we change some of its terms to 0 , this does not affect its convergence. Thus, from Theorem 5 it follows that $\left(a_{n}\right)_{n \in \mathbb{N}}$ converges to 0 if and only if $\left\|a_{n}\right\| \rightarrow 0$, and hence, by the remarks in the beginning of Section 4, we see that the new metric $d$ also generates the Furstenberg topology on $\mathbb{Z}$.

\subsection{Uniformly equivalent metrics}

If $(X, d)$ is a metric space, then there exists a complete metric space $(\bar{X}, d)$ which contains $X$ as a dense subspace. This complete metric space is uniquely determined up to an isometry, and it is called the completion of $(X, d)$. In the next subsection we shall construct the completion of the metric space $(\mathbb{Z}, d)$. Thus the following question arises naturally: if a topology on a set is induced by two (or more) different metrics, is there any connection between the completion of these metric spaces? In general, the answer is no. For example, the interval ]0,1[ is homeomorphic to $\mathbb{R}$ as a topological space. Their completions are $[0,1]$ and $\mathbb{R}$, which are, of course, not homeomorphic.

However, the completion that we shall describe is still a natural construction in a certain sense. The point is this. If $d_{1}$ and $d_{2}$ are two translation invariant metrics on $\mathbb{Z}$ which induce the same topology, e.g., the Furstenberg topology, then they are automatically uniformly equivalent, i.e., the identity mapping between $\left(\mathbb{Z}, d_{1}\right)$ and $\left(\mathbb{Z}, d_{2}\right)$ is uniformly continuous in both directions.

To see this, let \|\|$_{1}$ and \|\|$_{2}$ be the two norms inducing $d_{1}$ and $d_{2}$, respectively. If $\varepsilon>0$, then, since $d_{1}$ and $d_{2}$ are homeomorphic to each other, there is a number $\delta>0$ such that $\|n\|_{1}<\delta$ implies $\|n\|_{2}<\varepsilon$. Thus $d_{1}(m, n)=\|m-n\|_{1}<\delta$ implies $d_{2}(m, n)=$ $\|m-n\|_{2}<\varepsilon$, which shows that the identity mapping from $\left(\mathbb{Z}, d_{1}\right)$ to $\left(\mathbb{Z}, d_{2}\right)$ is uniformly continuous. Interchanging the role of $d_{1}$ and $d_{2}$, we obtain the assertion.

So if two translation invariant metrics induce the Furstenberg topology, then they are uniformly equivalent. The next theorem shows that the completion of $\mathbb{Z}$ endowed with such a metric is uniquely determined up to uniform equivalence.

Theorem 6. If $\left(X, d_{X}\right)$ and $\left(Y, d_{Y}\right)$ are uniformly equivalent metric spaces, then their completions are uniformly equivalent as well.

Proof. For simplicity, we shall denote the two metrics $d_{X}$ and $d_{Y}$ by the same symbol $d$, since this cannot cause any ambiguity. Let $f: X \rightarrow Y$ be a uniform equivalence, i.e., a bijective mapping between $X$ and $Y$ such that both $f$ and $f^{-1}$ are uniformly continuous, and let $(\bar{X}, d)$ and $(\bar{Y}, d)$ be the completion of $X$ and $Y$, respectively. 
We extend $f$ to a mapping $\tilde{f}: \bar{X} \rightarrow \bar{Y}$ in the following way. If $p \in \bar{X}$, then choose a sequence $\left(p_{n}\right)_{n \in \mathbb{N}}$ in $X$ converging to $p$. Since $f$ is uniformly continuous, $\left(f\left(p_{n}\right)\right)_{n \in \mathbb{N}}$ is a Cauchy sequence, and by the completeness of $\bar{Y}$, it is convergent in $\bar{Y}$. Let $\tilde{f}(p):=$ $\lim _{n \rightarrow \infty} f\left(p_{n}\right)$. It can be easily seen that $\tilde{f}(p)$ does not depend on the choice of the sequence $\left(p_{n}\right)_{n \in \mathbb{N}}$, and that $\tilde{f}$ is an extension of $f$.

Now we show that $\tilde{f}$ is uniformly continuous. Let $\varepsilon>0$ be arbitrary. By the uniform continuity of $f$, there exists $\delta>0$ such that $p, q \in X, d(p, q)<3 \delta$ implies $d(f(p), f(q))<$ $\varepsilon / 3$. Now let $p, q \in \bar{X}$ with $d(p, q)<\delta$, and let $\left(p_{n}\right)_{n \in \mathbb{N}}$ and $\left(q_{n}\right)_{n \in \mathbb{N}}$ be two sequences in $X$ converging to $p$ and $q$, respectively. Since they are Cauchy sequences as well, there is an index $n_{0} \in \mathbb{N}$ such that $d\left(p_{n}, p_{m}\right)<\delta$ and $d\left(q_{n}, q_{m}\right)<\delta$ whenever $n, m \geq n_{0}$, thus also $d\left(f\left(p_{n}\right), f\left(p_{m}\right)\right)<\varepsilon / 3$ and $d\left(f\left(q_{n}\right), f\left(q_{m}\right)\right)<\varepsilon / 3$. From these, taking the limit $m \rightarrow \infty$ we obtain

$$
d\left(p_{n}, p\right) \leq \delta, d\left(q_{n}, q\right) \leq \delta, d\left(f\left(p_{n}\right), \tilde{f}(p)\right) \leq \frac{\varepsilon}{3}, d\left(f\left(q_{n}\right), \tilde{f}(q)\right) \leq \frac{\varepsilon}{3} .
$$

Hence we have

$$
d\left(p_{n}, q_{n}\right) \leq d\left(p_{n}, p\right)+d(p, q)+d\left(q, q_{n}\right)<\delta+\delta+\delta=3 \delta .
$$

By the choice of $\delta$ this implies $d\left(f\left(p_{n}\right), f\left(q_{n}\right)\right)<\varepsilon / 3$, and finally,

$$
d(\tilde{f}(p), \tilde{f}(q)) \leq d\left(\tilde{f}(p), f\left(p_{n}\right)\right)+d\left(f\left(p_{n}\right), f\left(q_{n}\right)\right)+d\left(f\left(q_{n}\right), \tilde{f}(q)\right)<3 \cdot \frac{\varepsilon}{3}=\varepsilon
$$

which means that $\tilde{f}$ is uniformly continuous.

In the same way we extend $f^{-1}: Y \rightarrow X$ to a uniformly continuous mapping $\widetilde{f^{-1}}: \bar{Y} \rightarrow$ $\bar{X}$. If $p \in \bar{X}$, and $\left(p_{n}\right)_{n \in \mathbb{N}}$ is a sequence in $X$ converging to $p$, then $\left(f\left(p_{n}\right)\right)_{n \in \mathbb{N}}$ converges to $\tilde{f}(p)$, and, by the construction of $\widetilde{f^{-1}}$,

$$
p=\lim _{n \rightarrow \infty} p_{n}=\lim _{n \rightarrow \infty} f^{-1}\left(f\left(p_{n}\right)\right)=\widetilde{f^{-1}}(\tilde{f}(p)) .
$$

Interchanging the role of $\tilde{f}$ and $\widetilde{f^{-1}}$, we see that they are inverses to each other, thus $\tilde{f}$ is indeed a uniform equivalence between $\bar{X}$ and $\bar{Y}$.

\subsection{The completion of $(\mathbb{Z}, d)$}

We now turn to the completeness question of the metric space $(\mathbb{Z}, d)$, where the metric $d$ is defined by (2). It can be easily seen that this metric space is not complete, i.e., not every Cauchy sequence is convergent in the set $\mathbb{Z}$. For instance, let

$$
a_{n}=1 \cdot 1 !+1 \cdot 2 !+\cdots+1 \cdot n ! \text {. }
$$

Then $m$ ! $\mid\left(a_{n}-a_{m}\right)$ if $n \geq m$, hence $d\left(a_{n}, a_{m}\right) \leq \frac{1}{m}$, and $\left(a_{n}\right)_{n \in \mathbb{N}}$ is a Cauchy sequence. However, if a positive number was the limit of $\left(a_{n}\right)_{n \in \mathbb{N}}$, then all of its FNS digits would be 1 , which is obviously impossible. Clearly, $\left(a_{n}\right)_{n \in \mathbb{N}}$ does not converge to 0 , and, from 
the generalization of the factorial number system to negative numbers below, we shall see that it does not converge to a negative number either.

This simple consideration shows that we have to add additional 'numbers' to the set $\mathbb{Z}$ to make the above Cauchy sequence - and others - convergent. To this end, we generalize the factorial number system to negative integers. First we need a recursion that gives the FNS digits of a positive integer in an explicit manner.

Lemma 1. Suppose that $z$ is a positive integer, and consider the following recursion. Let $i_{1}$ be the remainder of $z$ divided by 2 . If $i_{1}, \ldots, i_{n-1}$ are already defined, then let $i_{n}$ be the remainder of

$$
\frac{z-i_{1} \cdot 1 !-\cdots-i_{n-1}(n-1) !}{n !}
$$

divided by $n+1$. Then there is a greatest index $m$ such that $i_{m} \neq 0$, and the FNS representation of $z$ is

$$
z=i_{1} \cdot 1 !+\cdots+i_{m} \cdot m !
$$

Proof. We start from the FNS representation of $z$, and we show that each digit $i_{n}$ satisfies the given recursion. For $n=1$ this is obvious. If $n \geq 2$, then

$$
\begin{aligned}
\frac{z-i_{1} \cdot 1 !-\cdots-i_{n-1}(n-1) !}{n !} & =\frac{i_{n} \cdot n !+\cdots+i_{m} \cdot m !}{n !} \\
& =i_{n}+i_{n+1} \frac{(n+1) !}{n !}+\cdots+i_{m} \frac{m !}{n !} .
\end{aligned}
$$

On the right-hand side, each term is divisible by $n+1$, except, possibly, for the first one, thus the remainder in the $n$th step of the recursion is indeed $i_{n}$, the $n$th FNS digit of $z$. Finally, if $m$ is the index of the greatest nonzero digit of $z$, then the recursion obviously gives $i_{n}=0$ for $n>m$.

Now if $z$ is a negative integer, then we modify this recursion in such a way that in every step we take the least nonnegative remainder. Thus, we let $i_{1}$ be the least nonnegative remainder of $z$ divided by 2 , and if $i_{1}, \ldots, i_{n-1}$ are already defined, then let $i_{n}$ be the least nonnegative remainder of

$$
\frac{z-i_{1} \cdot 1 !-\cdots-i_{n-1}(n-1) !}{n !}
$$

divided by $n+1$. For example, if we apply this procedure to the number -349 , then we obtain the sequence

$$
1,2,1,0,3,6,7,8,9,10, \ldots
$$

In this case we see that there is no greatest index $m$ such that $i_{m} \neq 0$. On the other hand, it can be easily seen that $z-i_{1} \cdot 1 !-\cdots-i_{n-1}(n-1)$ ! is the greatest among the multiples of $n$ ! which are not greater than $z$, just as in the case of $z$ positive. Thus, if $n$ is such that $n ! \geq|z|$, then

$$
\frac{z-i_{1} \cdot 1 !-\cdots-i_{n-1}(n-1) !}{n !}=\frac{-n !}{n !}=-1,
$$


whose greatest nonnegative remainder divided by $n+1$ is $i_{n}=n$. In conclusion, whereas for $z$ nonnegative we have $i_{n}=0$ if $n$ is large enough, for a negative $z$ we have $i_{n}=n$ if $n$ is large enough, as in the example above.

Thus every integer has a unique representation in terms of the factorial number system, and, as we have just seen, $z \geq 0$ if and only if there is an index $m$ such that $i_{n}=0$ for all $n>m$, and $z<0$ if and only if there is an index $m$ such that $i_{n}=n$ for all $n>m$.

Using the notation

$$
\mathbb{Z}_{n}:=\{0,1, \ldots, n-1\}
$$

we define

$$
\overline{\mathbb{Z}}:=\prod_{n=2}^{\infty} \mathbb{Z}_{n} .
$$

If we identify each integer with its FNS representation, then we may write $\mathbb{Z} \subset \overline{\mathbb{Z}}$. Now we extend the norm and metric on $\mathbb{Z}$ to $\overline{\mathbb{Z}}$. If $z=\left(i_{1}, i_{2}, i_{3}, \ldots\right) \in \overline{\mathbb{Z}}$, where $i_{n} \in \mathbb{Z}_{n+1}$ for each $n$, and at least one $i_{n}$ is different from zero, then let

$$
\|z\|:=\frac{1}{\min \left\{j \in \mathbb{N}: i_{j} \neq 0\right\}},
$$

and if $z=(0,0,0, \ldots)$, then $\|z\|:=0$. To introduce the metric on $\overline{\mathbb{Z}}$, we have to define a subtraction on $\overline{\mathbb{Z}}$ in such a way that it is consistent with subtraction in the FNS.

Lemma 2. Let $z_{1}=\left(i_{1}, i_{2}, i_{3}, \ldots\right)$ and $z_{2}=\left(j_{1}, j_{2}, j_{3}, \ldots\right)$ be two integers, and define the sequences $\left(k_{n}\right)_{n \in \mathbb{N}}$ and $\left(l_{n}\right)_{n \in \mathbb{N}}$ by the following recursion:

$$
\begin{aligned}
k_{1} & :=\left\{\begin{array}{ll}
0 & \text { if } i_{1}=j_{1}, \\
1 & \text { if } i_{1} \neq j_{1},
\end{array} \quad l_{1}:=\left\{\begin{array}{l}
1 \quad \text { if } i_{1}=0, j_{1}=1, \\
0 \quad \text { otherwise, }
\end{array}\right.\right. \\
k_{n} & := \begin{cases}i_{n}-j_{n}-l_{n-1} & \text { if } i_{n}-j_{n}-l_{n-1} \geq 0, \\
i_{n}-j_{n}-l_{n-1}+n+1 & \text { if } i_{n}-j_{n}-l_{n-1}<0,\end{cases} \\
l_{n} & := \begin{cases}0 & \text { if } i_{n}-j_{n}-l_{n-1} \geq 0, \\
1 & \text { if } i_{n}-j_{n}-l_{n-1}<0 .\end{cases}
\end{aligned}
$$

Then we have $z_{1}-z_{2}=\left(k_{1}, k_{2}, k_{3}, \ldots\right)$.

Proof. We shall show by induction that the $n$th FNS digit of $z_{1}-z_{2}$ is $k_{n}$ for each $n \in \mathbb{N}$. By the construction of the FNS digits described above, we have to show that $k_{1}$ is the least nonnegative remainder of $z_{1}-z_{2}$ divided by 2 , and $k_{n}$ is the least nonnegative remainder of $\left(z_{1}-z_{2}-k_{1} \cdot 1\right.$ ! $\left.-\cdots-k_{n-1} \cdot(n-1) !\right) / n$ ! divided by $n+1$, for each $n \geq 2$. The first assertion is obvious. Now suppose that $n \geq 2$, and $k_{1}, \ldots, k_{n-1}$ are the first $n-1$ digits of $z_{1}-z_{2}$. First observe that the inequalities $0 \leq i_{n} \leq n, 0 \leq j_{n} \leq n$ and $0 \leq l_{n-1} \leq 1$ imply

$$
-n-1 \leq i_{n}-j_{n}-l_{n-1} \leq n,
$$

from which it follows that $0 \leq k_{n} \leq n$ in either cases in the definition of $k_{n}$. Thus it remains to show that

$$
n+1 \mid \frac{z_{1}-z_{2}-k_{1} \cdot 1 !-\cdots-k_{n-1} \cdot(n-1) !}{n !}-k_{n},
$$


which is equivalent to

$$
(n+1) ! \mid z_{1}-z_{2}-k_{1} \cdot 1 !-\cdots-k_{n} \cdot n ! .
$$

By the definition of $i_{n}$ and $j_{n}$, we have

$$
\begin{aligned}
& (n+1) ! \mid z_{1}-i_{1} \cdot 1 !-\cdots-i_{n} \cdot n ! \\
& (n+1) ! \mid z_{2}-j_{1} \cdot 1 !-\cdots-j_{n} \cdot n !
\end{aligned}
$$

thus (3) is equivalent to

$$
(n+1) ! \mid\left(k_{1}-i_{1}+j_{1}\right) 1 !+\cdots+\left(k_{n}-i_{n}+j_{n}\right) n ! .
$$

Now it can be shown by a simple induction that

$$
\sum_{m=1}^{n}\left(k_{m}-i_{m}+j_{m}\right) m != \begin{cases}0 & \text { if } l_{n}=0 \\ (n+1) ! & \text { if } l_{n}=1 .\end{cases}
$$

From this, relations (4) and (3) follow at once, which verifies the assertion of the lemma.

If $z_{1}=\left(i_{1}, i_{2}, i_{3}, \ldots\right)$ and $z_{2}=\left(j_{1}, j_{2}, j_{3}, \ldots\right)$ are two points in $\overline{\mathbb{Z}}$, then define the sequences $\left(k_{n}\right)_{n \in \mathbb{N}}$ and $\left(l_{n}\right)_{n \in \mathbb{N}}$ by the same recursion as in Lemma 2, and finally let $z_{1}$ $z_{2}:=\left(k_{1}, k_{2}, k_{3}, \ldots\right)$. (It can be seen from the proof of the lemma that the sequence $\left(l_{n}\right)_{n \in \mathbb{N}}$ consists of merely 'auxiliary' digits, which do not appear in the final result of the subtraction.) Now let

$$
d: \overline{\mathbb{Z}} \times \overline{\mathbb{Z}} \rightarrow \mathbb{R}, d\left(z_{1}, z_{2}\right):=\left\|z_{1}-z_{2}\right\|
$$

Thus $\overline{\mathbb{Z}}$ becomes a metric space, and $\mathbb{Z}$ is its metric subspace.

Theorem 7. The metric space $(\overline{\mathbb{Z}}, d)$ is the completion of $(\mathbb{Z}, d)$.

Proof. We have to show that $(\overline{\mathbb{Z}}, d)$ is a complete metric space and that $\mathbb{Z}$ is dense in $\overline{\mathbb{Z}}$. Let $\left(a_{n}\right)_{n \in \mathbb{N}}$ be a Cauchy sequence in $\overline{\mathbb{Z}}$. Thus, for every $k \in \mathbb{N}$ there is an index $n_{0}$ such that $\left\|a_{n}-a_{m}\right\|<\frac{1}{k}$ if $n, m>n_{0}$, therefore the first $k$ components of $a_{n}-a_{m}$ are zero. As it can be seen from the algorithm of subtraction, this also implies that the first $k$ components of $a_{n}$ and $a_{m}$ are identical. Thus we may define $i_{k}$ to be the $k$ th component of $a_{n}$ with an arbitrary $n>n_{0}$. Now it is obvious from the construction that the sequence $\left(a_{n}\right)_{n \in \mathbb{N}}$ converges to $a:=\left(i_{1}, i_{2}, i_{3}, \ldots\right)$. Thus $(\overline{\mathbb{Z}}, d)$ is a complete metric space.

Finally, let $a=\left(i_{1}, i_{2}, i_{3}, \ldots\right)$ be an arbitrary point in $\overline{\mathbb{Z}}$ and $\varepsilon>0$. Choose a $k \in \mathbb{N}$ such that $\frac{1}{k}<\varepsilon$ and let $b:=\left(i_{1}, \ldots, i_{k}, 0, \ldots\right)$. Then $b \in \mathbb{Z}$ and $d(a, b)=\|a-b\|<\varepsilon$, which shows that $\mathbb{Z}$ is dense in $\overline{\mathbb{Z}}$. 


\section{Acknowledgement}

The first author is grateful to Professor Zsolt Páles for useful discussions on several points of this paper. The second author is grateful to Dr. Gábor Nyul, who originally introduced the Furstenberg topological space to him.

\section{References}

[1] M. Aigner, G.M. Ziegler, Proofs from the book, Springer-Verlag, 1998.

[2] K.A. Broughan, Adic topologies for the rational integers, Canad. J. Math. 55(4) (2003), 711-723.

[3] K.A. Broughan, F. Luca, On the Furstenberg closure of a class of binary recurrences, J. Number Th. 130(3) (2010), 696-706.

[4] A.J. Engler, A. Prestel, Valued fields, Springer-Verlag, 2005.

[5] J. Ferry, Science Forum Index, http://www.groupsrv.com/science/about483222.html

[6] H. Furstenberg, On the infinitude of primes, Amer. Math. Monthly, 62(5) (1955), 353.

[7] J.J. Gutiérrez G., E.A. Acevedo S., Metrizaciones y algunas propiedades de la topología de Fürstenberg, Tecnociencia 13(2) (2011), 67-80.

[8] S. Hernández, F. Luca, On a question of Broughan, Proc. Amer. Math. Soc. 136 (2008), 403-407.

[9] D.E. Knuth, The Art of Computer Programming Vol. 2., Addison-Wesley, Reading, 1997.

[10] R.L. Lovas, I. Mező, On an exotic topology of the integers, ArXiv, http://arxiv.org/abs/1008.0713.

[11] I.D. Mercer, On Furstenberg's proof of the infinitude of primes, Amer. Math. Monthly, 116 (2009), 355 356

[12] J. Munkres, Topology, Prentice Hall, 1975.

[13] L.A. Steen, J.A. Seebach, Counterexamples in topology, Dover, 1995.

[14] P. Szczuka, The connectedness of arithmetic progressions in Furstenberg's, Golomb's and Kirch's topologies, Dem. Math. 43(4) 2010, 899-909.

Rezső L. Lovas ${ }^{1}$

Institute of Mathematics

University of Debrecen

Debrecen, Hungary

e-mail: lovasescience.unideb.hu

István Mező ${ }^{2}$

Department of Mathematics

Nanjing University of Information Science \& Technology

Nanjing, 210044, P. R. China

e-mail: istvanmezo81@gmail.com

\footnotetext{
${ }^{1}$ Rezső L. Lovas was supported by the OTKA project K-111651 (Hungary).

${ }^{2}$ The research of István Mezô was supported by the Scientific Research Foundation of Nanjing University of Information Science \& Technology
} 